\title{
Exclusive Higgs boson production at the LHC: hard rescattering corrections
}

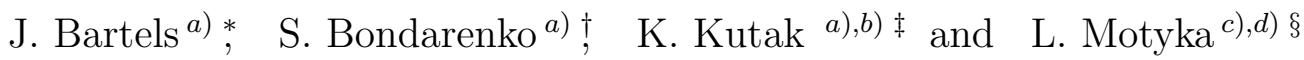 \\ a) II Institute for Theoretical Physics, University of Hamburg, Germany \\ b) H. Niewodniczański Institute of Nuclear Physics, Kraków, Poland \\ c) DESY Theory Group, Hamburg, Germany \\ d) Institute of Physics, Jagellonian University, Kraków, Poland
}

January 16, 2006

\begin{abstract}
We examine, as a correction to the central exclusive Higgs boson production in $p p$ collisions at the LHC, the rescattering of gluonic ladders off the proton. As usual, at the lowest order the hard part of this process can be described as a fusion of two hard gluonic ladders. We calculate corrections to this hard amplitude which are due to rescattering of these ladders. These corrections, which contain high mass diffractive excitations of the proton, have not yet been taken into account by the usual soft survival probabilities. We find that the correction due to the exchange of a single hard rescattering is negative, large and infrared sensitive. As a first step towards a more reliable description we therefore replace the rescattering exchange by a unitarized amplitude using the BK equation which generates the saturation scale $Q_{s}(x)$. We also include a soft gap survival probability factor. We discuss the results and outline possible future strategies.
\end{abstract}

\footnotetext{
*Email: bartels@mail.desy.de

${ }^{\dagger}$ Email: sergb@mail.desy.de

${ }^{\ddagger}$ E-mail: kutak@mail.desy.de

${ }^{\S}$ E-mail: motyka@th.if.uj.edu.pl
} 


\section{Introduction}

One of the main experimental goals to be achieved at the Large Hadron Collider (LHC) is the discovery of the Higgs boson and the investigation of its properties. The discovery potential of LHC should be sufficient to find the Standard Model Higgs boson as well as the supersymmetric Higgs particles in the wide range of masses, starting from the lower limit of $114.4 \mathrm{GeV}$ imposed by the LEP [1] data up to the mass of a few hundred GeV. Precise electroweak measurements indicate, however, that the mass of the Higgs boson should be rather at the lower end of the allowed window, with the central value at $113 \mathrm{GeV}$ being determined from radiative corrections, and the upper limit of about $240 \mathrm{GeV}$ at the confidence level of 95\%. It has been proposed [2]-15] that, if the Higgs boson mass, $M_{H}$, is close to the expected central value, a measurement of the exclusive diffractive reaction should be possible:

$$
p p \longrightarrow p H p
$$

with the Higgs boson separated by rapidity gaps from the protons. The main advantage of this process is an extremely low background which is much smaller than the potential signal. The background suppression permits precise measurements - for instance of the quantum numbers of the Higgs boson. Besides that, in the exclusive production process, detection of protons provides a complete information on the invariant mass of the produced system. This opens an exciting possibility to find resonances corresponding to new heavy particles that would be invisible in conventional measurements, see [16. 17. 18. The main drawback of the exclusive Higgs production channel, however, is its very low rate. The requirement of leaving the protons intact and of producing no secondary particles reduces the cross section by many orders of magnitude. In the series of papers [5]-[11] the authors discussed extensively the theoretical and phenomenological aspects of the exclusive production of heavy particle in $p p$ collisions in perturbative QCD, and their approach will be used as the basis of our study.

The mechanism of the considered process is illustrated in the diagram in in Fig. 10. Thus, the hard subprocess is given by a fusion of two colour singlet gluonic ladders into the Higgs scalar. The diagram may be evaluated in the $k_{t}$-factorisation framework using the unintegrated gluon densities. The required absence of hard radiation leaves un-compensated the virtual corrections to the process. Those corrections turn out to be large as they contain the scale of the Higgs boson mass generating large double and single logarithmic contributions. The necessary resummation of these logarithmically enhanced terms is realized by including the Sudakov form factor, which not only reduces the cross section but also provides the infrared stability of the hard exclusive scattering amplitude. Unfortunately, the basic hard subprocess is accompanied by multiple soft rescattering, which, typically, is inelastic and suppresses the exclusive cross section. Currently, the soft inelastic rescattering may be estimated only in phenomenological models [19, 20, 21, 22]. Present calculations of the gap survival probability rely predominantly on a two channel eikonal model for the $p p$ opacity, incorporating effects of the low mass diffraction and of the pionic cloud. The model was developed in Refs. 20, 21, and the 
a)

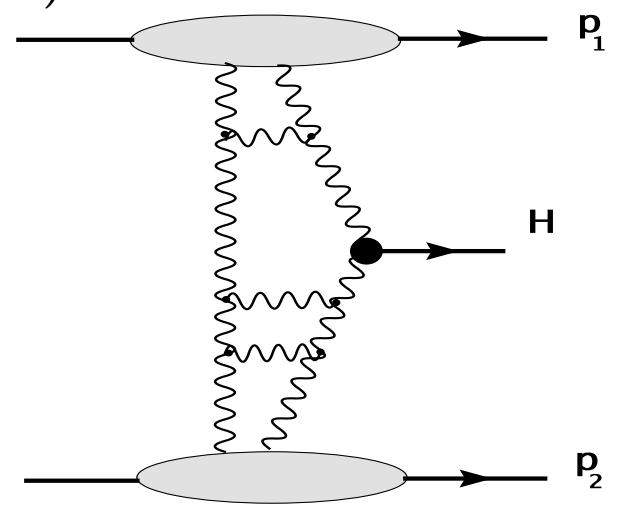

b)

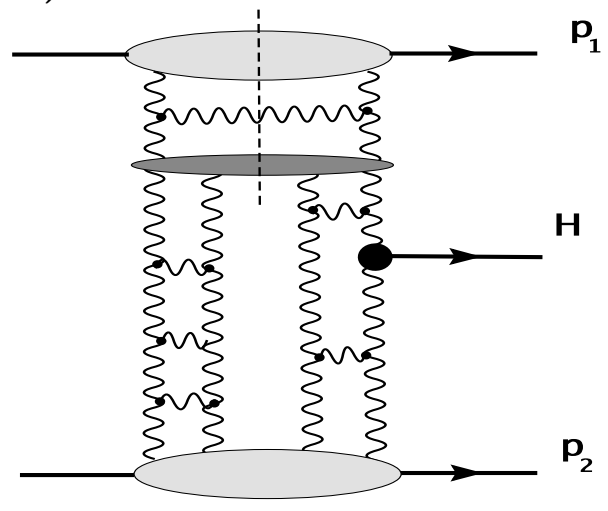

Figure 1: The exclusive Higgs boson production in a pp collision: a) the Two Pomeron Fusion contribution and b) a hard absorptive correction.

parameters were fitted to describe the data on the total $p p$ and $p \bar{p}$ cross section and on the elastic $p p$ and $p \bar{p}$ scattering.

In this paper we shall address the problem of computing a hard absorptive correction to the exclusive production process, which has already been mentioned in the literature [18 but has not been studied in detail yet. In brief, this contribution comes from an absorption of the hard gluonic ladders that fuse to produce the Higgs boson in the $p p$ scattering process, see Fig. 1p. This contribution can also be viewed as the rescattering of a large mass intermediate diffractive state moving along one of the protons (the proton at the top of in Fig. 1b; let us call it the projectile) off the other proton (the proton at the bottom of in Fig. 10, the target), and this contribution is not part of the soft rescattering of the spectators that contributes to the standard opacity. In Fig. 10, the large mass diffractive state is indicated by a dashed line. In perturbative QCD, within the leading logarithmic $1 / x$ approximation, the rescattering is driven by the exchange of a BFKL Pomeron [23, 24, 25, 26] which couples to the hard gluonic ladder by the triple Pomeron vertex [27, 28, 29, 30. Such a contribution is potentially large, since the BFKL rapidity evolution between the target proton and the triple Pomeron vertex extends over the length of $Y>14$ and thus may generate an enhancement by two orders of magnitude. If this correction is really as large as anticipated, it is clear that also higher order unitarity corrections to this hard rescattering will be sizable and should be evaluated as well.

As we shall discuss in detail, the situation is even more complicated. Namely, when evaluating the rescattering correction based on a simple BFKL Pomeron exchange, it turns out that the amplitude - which is infrared finite - exhibits a high sensitivity to the details of the infrared region. To be more precise, the momentum scale around the triple Pomeron vertex tends to be small. At first sight, therefore, this naïve approach seems to be highly questionable. However, if we include - perturbative unitarity corrections, the problems with the infrared sensitivity may be greatly reduced. As a guiding example let as quote results of Refs. 34, 35] on the impact of a special class of unitarity corrections on 
the behaviour of the gluon distribution at low momenta. In these references the Balitsky-Kovchegov (BK) equation [31, 32, 33] was investigated, as a model which unitarizes the BFKL Pomeron exchange by a resummation of the BFKL Pomeron fan diagrams. It was found that the equation generates a momentum scale, the saturation scale $Q_{s}(Y)$, which provides an effective lower cut-off on the gluon momenta. The saturation scale grows exponentially with the evolution length $Y, Q_{s}(Y) \sim \exp (\lambda Y)$, with $\lambda \simeq 0.3$, and the saturation scale enters the region in which perturbative QCD is applicable if $Y$ is sufficiently large. Thus, in the presence of the perturbative saturation scale the high sensitivity to the infrared domain is eliminated. Let us stress here that the concept of a rapidity dependent saturation scale is rather robust. The concept has received strong support from phenomenological considerations, being the central part of the very fruitful saturation model [36, 37. It was also proven that the generation of the saturation scale is a universal phenomenon in a wide class of non-linear evolution equations 38, 39, 40, which gives some confidence that the emergence of the saturation scale is not an accidental feature of the BK equation.

In the second part of our investigation, therefore, we shall replace the linear BFKL equation for the screening Pomeron by the non-linear Balitsky-Kovchegov evolution equation. This corresponds to the resummation of the BFKL Pomeron fan diagrams originating from the target proton: this resummation will be shown to stabilize the correction amplitude in the infrared region. Certainly, in doing so we have made rather crude approximations. Namely, we have not taken into account other important unitarity corrections present in the case of proton-proton scattering, for instance those related to possible interactions of the Pomerons in the fan diagrams with the projectile proton (for a more detailed discussion see Section 7). For the total cross section of nucleus-nucleus collisions an effective field theory of interacting BFKL Pomerons has been formulated [41, 42, 43]. For $p p$ scattering an analogous framework is still to be developed. We therefore view our study only as a first step towards constructing a theoretical framework for evaluating hard absorptive corrections to the exclusive high mass production in $p p$ collisions. We shall compute the magnitude of the correction in this, rather crude, approximation in order to provide some insight into how important the discussed effects might be.

The structure of the paper is the following. In Section 2 we briefly review the standard framework introduced in the series of papers [5]-[11]. We derive the hard rescattering correction in Section 3. The calculation of the soft gap survival probability for the hard corrected amplitude is given in Section 4 . In Section 5 the Balitsky-Kovchegov evolution of the gluon density is described. The results of the numerical evaluation of the hard rescattering corrections and the obtained cross sections are presented in Section 6. Implications of the results and future strategies are discussed in Section 7. Finally, conclusions are presented in Section 8. 


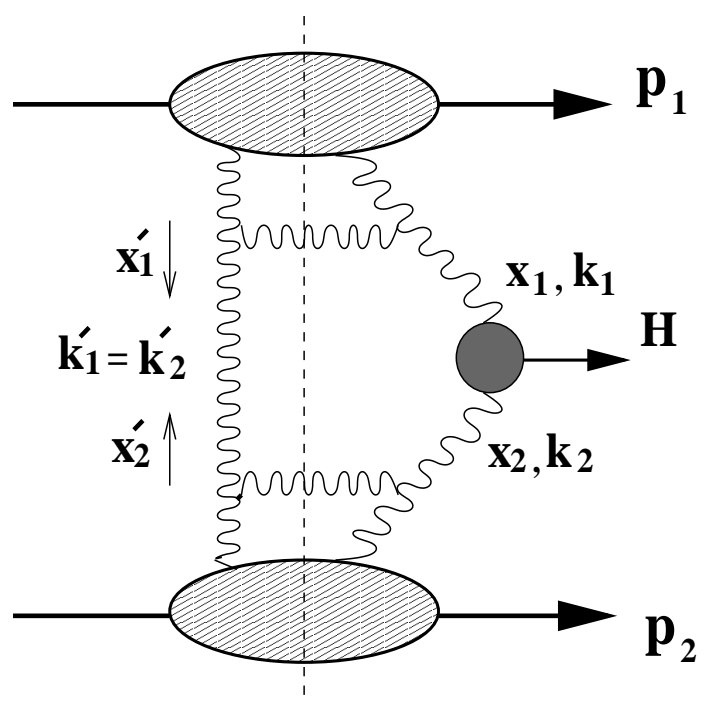

Figure 2: Kinematics of the Two Pomeron Fusion contribution to the exclusive Higgs boson production.

\section{The hard amplitude for exclusive Higgs production}

In this Section we briefly review the main features of the standard QCD approach [5]-[1] to the hard part of the exclusive Higgs boson production in $p p$ collisions.

The basic diagram is given in Fig. 2] the leading contribution of this amplitude is imaginary. The diagram is evaluated in the high energy limit, where the $t$-channel gluons are in the so-called nonsense polarisation states, and their virtualities are dominated by the transverse components of their momenta. The matrix element describing the fusion of two gluons into the Higgs boson is given by the top quark triangle, taken in the limit of a point-like coupling. This effective approximation is very accurate as the gluon virtualities are much smaller than the top quark mass. The amplitude of finding, in the proton, two gluons that carry tranverse momenta $\boldsymbol{k}$ and $\boldsymbol{k}^{\prime}$ and fractions $x$ and $x^{\prime}$ of the longitudinal momentum of the parent proton is represented by the off-diagonal unintegrated gluon density $f_{g}^{\text {off }}\left(x, x^{\prime} ; \boldsymbol{k}, \boldsymbol{k}^{\prime} ; \mu\right)$, assuming that the density is probed at the scale $\mu$. The three subamplitudes (Fig. 2), corresponding to the gluon emissions and to the Higgs boson production, are convoluted using the $k_{t}$-factorisation.

The kinematics of the exclusive Higgs boson production determines the arguments of the offdiagonal gluon densities. Thus, the elastic form-factor of the proton provides a sharp cut-off, which we assume to be Gaussian: $\sim \exp \left[-\left(\boldsymbol{k}-\boldsymbol{k}^{\prime}\right)^{2} R^{2} / 4\right]$ with $R^{2} \simeq 8 \mathrm{GeV}^{2}$, on the momentum transfer carried by the gluonic system. Therefore, it is appropriate to write

$$
f_{g}^{\text {off }}\left(x, x^{\prime} ; \boldsymbol{k}, \boldsymbol{k}^{\prime} ; \mu\right) \simeq f_{g}^{\text {off }}\left(x, x^{\prime} ; k^{2} ; \mu\right) \exp \left[-\left(\boldsymbol{k}-\boldsymbol{k}^{\prime}\right)^{2} R^{2} / 4\right] .
$$

For future use, let us also define the unintegrated gluon distribution depending on the position $\boldsymbol{b}$ in the transverse plane

$$
\tilde{f}_{g}^{\text {off }}\left(x, x^{\prime} ; k^{2}, \boldsymbol{b} ; \mu\right)=\int \frac{d^{2} q}{(2 \pi)^{2}} e^{i \boldsymbol{q} \boldsymbol{b}} f_{g}^{\text {off }}\left(x, x^{\prime} ; \boldsymbol{k}, \boldsymbol{q}-\boldsymbol{k} ; \mu\right),
$$


where $\boldsymbol{q}=\boldsymbol{k}-\boldsymbol{k}^{\prime}$

The next point that should be discussed is the disparity between the longitudinal momentum fractions $x$ and $x^{\prime}$. The production of the heavy scalar particle occurs close to the central region where $x \simeq M_{H} / \sqrt{s}$ and $x^{\prime} \sim \sqrt{k^{2} / s}$, so $x^{\prime} \ll x$. At the leading logarithmic level the off-diagonal (w.r.t. $x$ ) distribution reduces to the diagonal one. Beyond the LL accuracy the effect of different $x$ values of the gluons may be approximated by a constant factor [4]

$$
R_{\xi}=\frac{2^{2 \lambda+3}}{\sqrt{\pi}} \frac{\Gamma(\lambda+5 / 2)}{\Gamma(\lambda+4)}
$$

provided the diagonal gluon distribution has a power-like $x$-dependence, $f_{g}\left(x, k^{2} ; \mu\right) \sim x^{-\lambda}$. For moderate scales one has $\lambda \simeq 0.25$.

The last important point is the scale dependence of the unintegrated gluon density. In the BFKL approach to the evolution of the gluon density there is no dependence of the gluon density on the external scale. This happens because the virtual perturbative QCD corrections to the coupling of the ladder to the Higgs production vertex generate only the DGLAP $\operatorname{logarithms} \log \left(\mu^{2} / k^{2}\right)$ (double and single) and not the small $x$ logarithms. In double logarithmic (in $\log \left(\mu^{2} / k^{2}\right)$ ) accuracy, the large corrections may be resumed into the Sudakov form-factor [6]:

$$
T_{s}(k, \mu)=\exp \left(-\int_{k^{2}}^{\mu^{2}} \frac{d p^{2}}{p^{2}} \frac{N_{c} \alpha_{s}}{\pi} \int_{p}^{\mu} \frac{d \omega}{\omega}\right) .
$$

The calculation of the Sudakov form-factor at single logarithmic accuracy has also been performed [6], giving

$$
T_{g}(\boldsymbol{k}, \mu)=\exp \left(-\int_{k^{2}}^{\mu^{2}} \frac{d p^{2}}{p^{2}} \frac{\alpha_{s}\left(p^{2}\right)}{2 \pi} \int_{0}^{1-\delta} d z z\left[P_{g g}(z)+\sum_{q} P_{q g}(z)\right]\right),
$$

where $\delta=\frac{p}{p+\mu}$ is chosen to provide the correct angular ordering of the real gluon emissions, and $P_{g g}$ and $P_{q g}$ are the DGLAP leading order splitting functions. For the exclusive Higgs production the scale $\mu \simeq 0.62 M_{H}$ should be chosen [1] in order to match exactly the first order single logarithmic correction. In our calculation we shall adopt the scale $\mu=M_{H} / 2$, which does not introduce a significant change of the result compared to the choice of [11].

A thorough discussion of how to determine the two-scale diagonal unintegrated gluon distribution has been presented in Refs. [45, 46]. A simple and economic way to incorporate the Sudakov formfactor into the unintegrated gluon distribution is given by the approach in which the last step of the DGLAP evolution is resolved in terms of the transverse momentum. In this approach, the twoscale unintegrated gluon distribution is obtained from the collinear gluon distribution in the following way [47, 45, 46]:

$$
f_{g}\left(x, k^{2} ; \mu\right)=Q^{2} \frac{\partial}{\partial Q^{2}}\left[x g\left(x, Q^{2}\right) \cdot T_{g}(Q, \mu)\right]_{Q^{2}=k^{2}} .
$$

In the off-diagonal case which is relevant for our present study, the logarithmic virtual corrections are associated with the production vertex at which the pair of gluons merges (see Fig. 2), in contrast to 
the standard case of a diagonal gluon distribution in DIS or in a hard inclusive production processes. Thus, in (7) the Sudakov form-factor has to be replaced by its square root.

Collecting all these results we arrive at:

$$
f_{g}^{\text {off }}\left(x, k^{2} ; \mu\right)=R_{\xi} Q^{2} \frac{\partial}{\partial Q^{2}}\left[x g\left(x, Q^{2}\right) \cdot \sqrt{T_{g}(Q, \mu)}\right]_{Q^{2}=k^{2}} .
$$

After contracting the polarisation tensors of the gluons with the $g g H$ vertex we obtain the dominant imaginary part of the hard exclusive amplitude for $p p \rightarrow p H p$ in the forward direction in the following form:

$$
\operatorname{Im} M_{0}(y)=2 \pi^{3} A \int \frac{d^{2} k}{2 \pi k^{4}} f_{g}^{\text {off }}\left(x_{1}, k^{2} ; \mu\right) f_{g}^{\text {off }}\left(x_{2}, k^{2} ; \mu\right) .
$$

Here the constant $A$ is given by

$$
A^{2}=K \sqrt{2} G_{F} \alpha_{S}^{2}\left(M_{H}^{2}\right) / 9 \pi^{2},
$$

where $G_{F}$ is the Fermi coupling, and $K \approx 1.5$ is the NLO $K$-factor. The Higgs boson rapidity in the centre of mass frame, $y$, defines the values of longitudinal momentum fractions:

$$
x_{1,2}=\frac{M_{H}}{\sqrt{s}} \exp ( \pm y)
$$

The differential hard cross-section following from this amplitude takes the form:

$$
\left.\frac{d \sigma_{p p \rightarrow p H p}(y)}{d y d t_{1} d t_{2}}\right|_{t_{1}=t_{2}=0}=\frac{\left|M_{0}(y)\right|^{2}}{256 \pi^{3}} .
$$

After the inclusion of the proton elastic form-factors in the non-forward direction (parameterized by $\sim \exp \left[-\left(\boldsymbol{k}-\boldsymbol{k}^{\prime}\right)^{2} R^{2} / 4\right]$ in (2)) one obtains the dependence of the cross section on $t_{1}$ and $t_{2}$ of the form of $\exp \left(R^{2}\left(t_{1}+t_{2}\right) / 2\right)$. Thus, after the integration over momentum transfers $t_{1}$ and $t_{2}$, the single differential cross section reads:

$$
\frac{d \sigma_{p p \rightarrow p H p}(y)}{d y}=\frac{\left|M_{0}(y)\right|^{2}}{64 \pi^{3} R^{4}}
$$

Note that, in (92), the form of the integral over the transverse momentum suggests an important sensitivity to the infrared domain. The rescue comes from the Sudakov form-factor which suppresses the low momenta of the unintegrated gluons faster than any power of $k$. It turns out that the dominant contribution to the integral (9) comes from momenta $k>1 \mathrm{GeV}$.

More details about the derivation of the amplitude of the exclusive Higgs boson production are given in the series of papers [5]-11]. In what follows we shall refer to the amplitude (9) as to the Two Pomeron Fusion (TPF) Amplitude. 


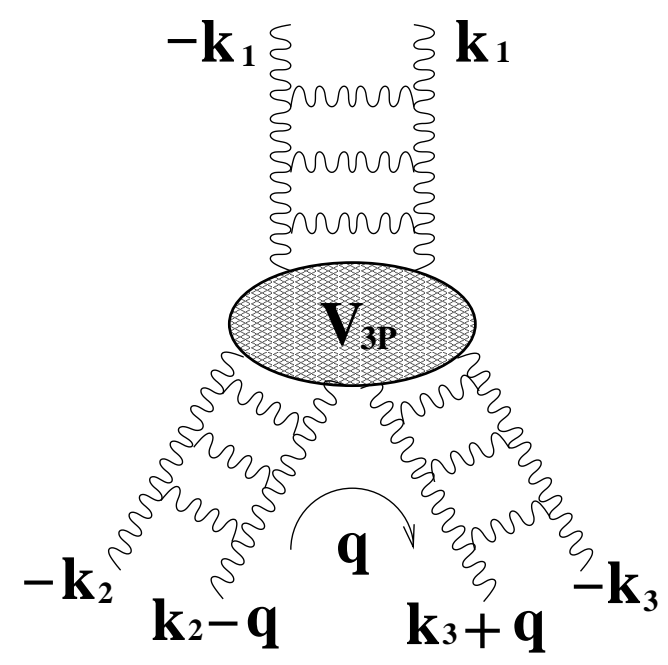

Figure 3: The triple Pomeron vertex.

\section{$3 \quad$ Hard rescattering corrections}

\subsection{Construction of the amplitude}

The amplitude (9) includes resummed QCD corrections up to single logarithms of the Higgs boson mass. The large available rapidity gap between the scattered protons ( $Y_{\text {tot }} \sim 19$ for the LHC energies), however, suggests that significant QCD corrections enhanced by small $x$ logarithms should be also considered. The spectator rescattering is expected to be of non-perturbative nature, and commonly it is accounted for by a soft gap survival probability factor.

Fig. [ illustrates a process of absorption of the perturbative gluonic ladder from the projectile (upper) proton in the target (lower) proton by the exchange of a QCD Pomeron across a large rapidity gap. Let us stress that the evolution length in rapidity of the screening Pomeron is large only if it couples to the ladder between the projectile proton and the produced Higgs boson. To be more specific, for the central Higgs boson production at the LHC the rapidity distance between the hard ladder that originates from one proton, and the other proton, $y_{4}=\log \left(1 / x_{4}\right)>14$, is large while the rapidity range occupied by each of the two hard ladders, is much smaller, $y_{1}=\log \left(1 / x_{1}\right)<5$ and $y_{3}=\log \left(1 / x_{3}\right)<5$. In contrast to this, the rapidity distance $y_{4}$ can be much larger, and a small $x$ (BFKL [26]) resummation of the perturbative QCD series may lead to a large enhancement of the amplitude $\sim \exp \left(\lambda y_{4}\right)$ with $\lambda \simeq 0.3$. Therefore this correction may be sizable, despite the fact it is suppressed by the factor of $\alpha_{s}^{2}$ at the triple Pomeron vertex, illustrated in Fig. 3, Thus, we shall evaluate the absorptive correction corresponding to the rescattering of the hard projectile gluonic system off the target proton. The coupling of the screening Pomeron to the screened gluonic ladder is known in the high energy limit; it is derived from the triple Pomeron vertex [27, 28, 29, 30.

The triple Pomeron vertex in pQCD has been computed in the LL1/x approximation [27, 28], and it follows from the $2 \rightarrow 4$ gluon vertex by projecting on the colour singlet states of the diagram represented symbolically in Fig. 3. In the process of interest the momentum transfers of all three 


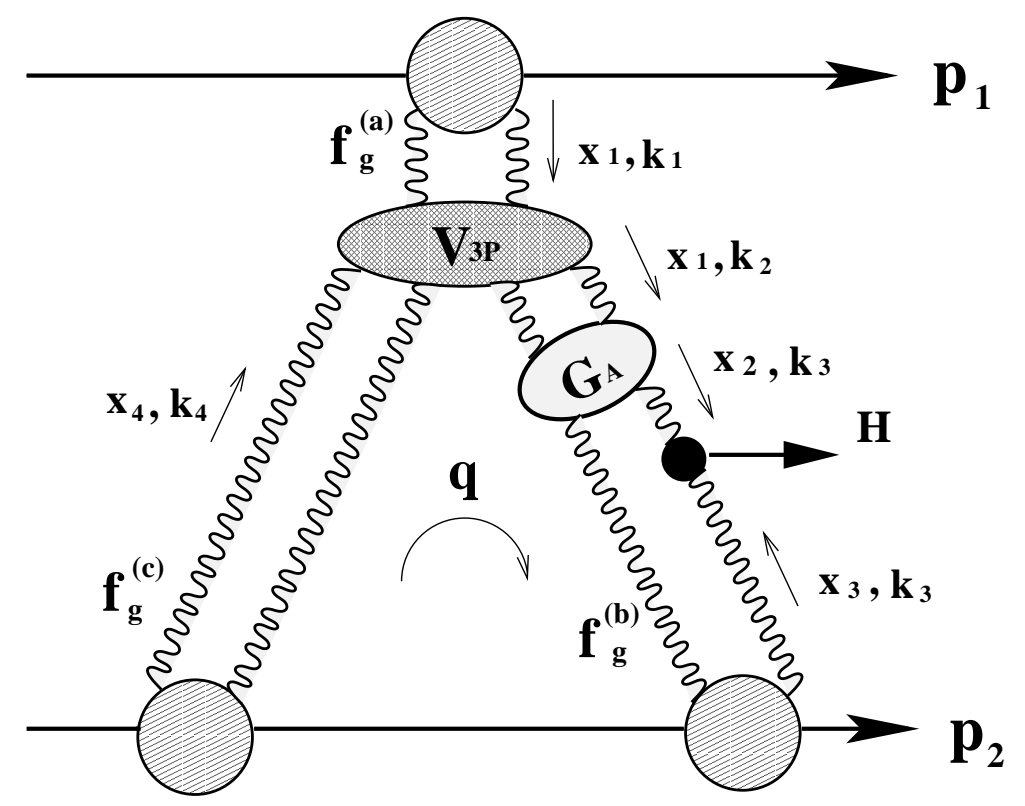

Figure 4: The hard rescattering correction to the exclusive Higgs boson production.

Pomerons are small: for the upper Pomeron and for the lower left one they are damped by the elastic proton form-factors. We, therefore, will neglect them in the expression for the triple Pomeron vertex, and it is will be sufficient to evaluate the vertex in the triple-forward limit. In this limit, the convolution $\left[V_{3 P} \otimes D\right]$ of the triple Pomeron vertex $V_{3 P}$ with the two-gluon amplitude above the vertex (here we follow the notation of [28, below we will replace $D$ by the unintegrated gluon density) is given by [27, 28]:

$$
\begin{aligned}
& {\left[V_{3 P} \otimes D\right]\left(\boldsymbol{k}_{2}, \boldsymbol{k}_{3}\right)=} \\
& \alpha_{s}^{2} c_{3 P} \int \frac{d^{2} \boldsymbol{k}_{1}}{2 \pi k_{1}^{2}}\left\{\left(\frac{k_{2}^{2}}{\left(\boldsymbol{k}_{1}-\boldsymbol{k}_{2}\right)^{2}}+\frac{k_{3}^{2}}{\left(\boldsymbol{k}_{1}+\boldsymbol{k}_{3}\right)^{2}}-\frac{\left(\boldsymbol{k}_{2}+\boldsymbol{k}_{3}\right)^{2} k_{1}^{2}}{\left(\boldsymbol{k}_{1}-\boldsymbol{k}_{2}\right)^{2}\left(\boldsymbol{k}_{1}+\boldsymbol{k}_{3}\right)^{2}}\right) D\left(k_{1}\right)\right. \\
& -\frac{k_{1}^{2}}{\left(\boldsymbol{k}_{1}-\boldsymbol{k}_{2}\right)^{2}}\left(\frac{k_{2}^{2}}{\left(\boldsymbol{k}_{1}-\boldsymbol{k}_{2}\right)^{2}+k_{1}^{2}}-\frac{\left(\boldsymbol{k}_{2}+\boldsymbol{k}_{3}\right)^{2}}{\left(\boldsymbol{k}_{1}-\boldsymbol{k}_{2}\right)^{2}+\left(\boldsymbol{k}_{1}+\boldsymbol{k}_{3}\right)^{2}}\right) D\left(k_{2}\right) \\
& \left.-\frac{k_{1}^{2}}{\left(\boldsymbol{k}_{1}+\boldsymbol{k}_{3}\right)^{2}}\left(\frac{k_{3}^{2}}{\left(\boldsymbol{k}_{1}+\boldsymbol{k}_{3}\right)^{2}+k_{1}^{2}}-\frac{\left(\boldsymbol{k}_{2}+\boldsymbol{k}_{3}\right)^{2}}{\left(\boldsymbol{k}_{1}-\boldsymbol{k}_{2}\right)^{2}+\left(\boldsymbol{k}_{1}+\boldsymbol{k}_{3}\right)^{2}}\right) D\left(k_{3}\right)\right\}
\end{aligned}
$$

where $\pm \boldsymbol{k}_{2}$ and $\pm \boldsymbol{k}_{3}$ are gluon momenta inside the two Pomerons below the vertex (see Fig. (3). Note that, in formula (14), the triple Pomeron vertex includes both real (the first term in the l.h.s.) and virtual (the next term in the l.h.s.) contributions. The normalisation factor, $c_{3 P}$, of the vertex will fixed by the form of the final expression for the correction amplitude (18), and we have constructed this expression in such a way that $c_{3 P}=1$.

In order to build up the complete expression of the amplitude of the diagram shown in Fig. 4 it is necessary to take into account the following building blocks:

1. The unintegrated gluon distribution, $f_{g}^{(a)}\left(x_{1}, k_{1}^{2} ; \mu_{a}\right)$, describing the amplitude of the gluons 
above the triple Pomeron vertex $V_{3 P}$. This gluon distribution is evaluated at relatively large $x_{1}$, and the momentum scale in the vertex should be given by the typical virtualities of the vertex, $\mu_{a} \simeq k_{1}$. So the gluon distribution will be evaluated using (17) $)^{1}$, and we shall denote the convolution (14) of the triple Pomeron vertex with the unintegrated gluon, $f_{g}^{(a)}\left(x_{1}, k_{1} ; k_{1}\right)$, by

$$
\left[V_{3 P} \otimes f_{g}^{(a)}\left(x_{1}\right)\right]\left(k_{2}, k_{3}\right)
$$

2. The two-gluon Green's function $G_{A}\left(\boldsymbol{k}_{2}, \boldsymbol{k}_{3} ; x_{1}, x_{2} ; \mu_{a}, \mu_{b}\right)$ that describes the propagation of the two gluon singlet between the Higgs production vertex and the triple Pomeron vertex. Two approximations for the Green's functions will be considered: the two gluon approximation $\left(G_{0}(\ldots)\right)$ and the diffusion approximation to the BFKL evolution $\left(G_{\mathrm{BFKL}}(\ldots)\right)$. The Green's function will have the typical scales $\mu_{a}$ and $\mu_{b}$, where the latter scale belongs to the Higgs production vertex. As to the scale of the triple Pomeron vertex, $\mu_{a} \sim k_{2}$, a priori, we do not know whether it will be large. However, the large scale is certainly present in the Higgs vertex, $\mu_{b} \simeq M_{H} / 2$. In order to account for the scale dependence at the Higgs vertex, the Sudakov form factor, $\sqrt{T_{g}\left(k_{3}, \mu_{b}\right)}$, will be included into the Green's function:

$$
G_{0}\left(\boldsymbol{k}_{2}, \boldsymbol{k}_{3} ; x_{1}, x_{2} ; \mu_{b}\right)=\sqrt{T_{g}\left(k_{3}, \mu_{b}\right)} k_{2}^{4} \delta^{(2)}\left(\boldsymbol{k}_{2}-\boldsymbol{k}_{3}\right)
$$

and

$$
G_{\mathrm{BFKL}}\left(\boldsymbol{k}_{2}, \boldsymbol{k}_{3} ; x_{1}, x_{2} ; \mu_{b}\right)=\frac{k_{2} k_{3}}{2 \pi} \frac{\sqrt{T_{g}\left(k_{3}, \mu_{b}\right)}}{\sqrt{\pi D \log \left(x_{1} / x_{2}\right)}} \exp \left[-\frac{\log ^{2}\left(k_{2}^{2} / k_{3}^{2}\right)}{4 D \log \left(x_{1} / x_{2}\right)}\right]\left(\frac{x_{1}}{x_{2}}\right)^{\omega_{0}},
$$

where only the leading conformal spin was taken into account, $\omega_{0}=4 \log (2) \bar{\alpha}_{s}$ is the LL BFKL intercept, $D=14 \zeta(3) \bar{\alpha}_{s}$ is the diffusion coefficient, and $\bar{\alpha}_{s}=3 \alpha_{s} / \pi$.

3. The Higgs boson production vertex which does not differ from the TPF case.

4. The off-diagonal unintegrated gluon distribution, $f_{g}^{(b)}\left(x_{3}, \boldsymbol{k}_{3}, \boldsymbol{q}-\boldsymbol{k}_{3} ; \mu_{b}\right)$, of the gluon distribution that enters the Higgs boson production vertex from the lower side, with the total transverse momentum $\boldsymbol{q}$. The gluon distribution is taken to be the same as in the case of the Two Pomeron Fusion, i.e. it is an off-diagonal gluon distribution given by formulae (8) and (2) and with $\mu_{b}=M_{H} / 2$.

5. The off-diagonal unintegrated gluon distribution, $f_{g}^{(c)}\left(x_{4}, \boldsymbol{k}_{4},-\boldsymbol{q}-\boldsymbol{k}_{4} ; \mu_{c}\right)$, for the screening Pomeron that propagates across the large rapidity distance from the lower proton to the triple Pomeron vertex with momentum transfer $\boldsymbol{- q}$. The scale $\mu_{c}$ is set by the gluon virtuality, so one has $f_{g}^{(c)}\left(x_{4}, \boldsymbol{k}_{4},-\boldsymbol{q}-\boldsymbol{k}_{4}\right)=f_{g}^{(c)}\left(x_{4}, \boldsymbol{k}_{4},-\boldsymbol{q}-\boldsymbol{k}_{4} ; k_{4}\right)$. For a detailed discussion of this gluon distribution see Section 4.

\footnotetext{
${ }^{1}$ Note that, for $\mu_{a}=k_{1}$, the Sudakov form factor, $T_{g}\left(k_{1}, \mu_{a}\right)$, in (7) introduces only a minor effect.
} 
After these definitions the expression for the dominant imaginary part of the diagram of Fig. [4 can be written in the following form:

$$
\begin{aligned}
\operatorname{Im} M_{\text {corr }}^{(1)}(y) & =-\frac{9}{8} 16 \pi^{2}\left(2 \pi^{3} A\right) \int_{x_{a}}^{x_{b}} \frac{d x_{4}}{x_{4}} \int \frac{d^{2} q}{(2 \pi)^{2}} \int \frac{d^{2} k_{2}}{2 \pi k_{2}^{4}} \int \frac{d^{2} k_{3}}{2 \pi k_{3}^{4}} \int \frac{d^{2} k_{4}}{2 \pi k_{4}^{4}} \\
& \times\left[V_{3 P} \otimes f_{g}^{(a)}\left(x_{1}\right)\right]\left(\boldsymbol{k}_{2}, \boldsymbol{k}_{4}\right) G_{\mathrm{A}}\left(\boldsymbol{k}_{2}, \boldsymbol{k}_{3} ; x_{1}, x_{2} ; M_{H} / 2\right) \\
& \times f_{g}^{(b)}\left(x_{3}, \boldsymbol{k}_{3}, \boldsymbol{q}-\boldsymbol{k}_{3} ; M_{H} / 2\right) f_{g}^{(c)}\left(x_{4}, \boldsymbol{k}_{4},-\boldsymbol{q}-\boldsymbol{k}_{4}\right),
\end{aligned}
$$

where $\frac{9}{8}=\frac{N_{c}^{2}}{N_{c}^{2}-1}$ tends to unity in the large $N_{c}$ limit, and the coefficient $2 \pi^{3} A$ has been extracted in order to enable an easier comparison with the Two Pomeron Fusion contribution given by (9). $x_{a}$ and $x_{b}$ denote the lower and the upper limits of the integration over $x_{4}$, respectively. The fractions of the longitudinal momenta of the upper proton $x_{1}$ and $x_{2}$ and of the lower proton $x_{3}, x_{4}$ read:

$$
\begin{gathered}
x_{3}=\frac{M_{H}}{\sqrt{s}} \exp (-y), \quad x_{2}=\frac{M_{H}}{\sqrt{s}} \exp (y), \quad x_{1} \simeq \frac{k_{0}^{2}}{x_{4} s} \\
x_{a}<x_{4}<x_{b}, \quad x_{a} \simeq \frac{k_{0}^{2}}{s}, \quad x_{b} \simeq \frac{k_{0}^{2}}{x_{2} s} .
\end{gathered}
$$

The energy scale $k_{0}^{2}$ that enters the definitions of gluon $x_{i}$ corresponds to typical values of the virtualities, and we set $k_{0}^{2}=1 \mathrm{GeV}^{2}$. The dependence on the momentum transfer $\boldsymbol{q}$, circulating in the Pomeron-proton triangle, appears only inside the off-shell unintegrated gluon distributions, $f_{g}^{(b)}$ and $f_{g}^{(c)}$, which contain the rapidly varying proton form-factor. The $\boldsymbol{q}$ dependence of the other vertices and of the Green's function, $G_{A}$, is weak and will be neglected. The amplitude (18) includes a factor of 2 coming from the possible emissions of the Higgs boson from both of the Pomerons below the triple Pomeron vertex. In addition, we have to add another contribution in which we interchange the role of projectile and target: since the structure of both protons is identical, we simply replace $y \rightarrow-y$. So finally the correction amplitude may be written as:

$$
M_{\text {corr }}(y)=M_{\text {corr }}^{(1)}(y)+M_{\text {corr }}^{(1)}(-y) \text {. }
$$

In the case of the central production, $y=0$, and $M_{\mathrm{corr}}(y)=2 M_{\mathrm{corr}}^{(1)}(y)$.

\subsection{Infrared stability}

It is instructive to study the infrared behaviour of the integral in (18) using a simplified framework and the saddle point method. First of all, we note that the integrals in (18) are infrared finite. This follows from properties of the triple Pomeron vertex (which vanishes whenever one of the external momenta goes to zero) and of the unintegrated gluon densities (which, as a result of gauge invariance, also vanish as one of the two gluon momenta goes to zero). Next, after averaging over the angles in $\boldsymbol{k}_{2}$ and $\boldsymbol{k}_{4}$, the triple Pomeron vertex (14) imposes the conditions $k_{2} \geq k_{1}$ and $k_{4} \geq k_{1}$. Finally, disregarding 
irrelevant constants and details one finds that, for the real contribution of the triple Pomeron vertex, the correction term (18) behaves as

$$
Q_{R} \sim \int_{k_{0}^{2}} \frac{d k_{1}^{2}}{k_{1}^{2}} \int_{k_{1}^{2}} \frac{d k_{2}^{2}}{k_{2}^{4}} \int_{k_{1}^{2}} \frac{d k_{4}^{2}}{k_{4}^{4}} f_{g}^{(a)}\left(x_{2}, k_{1}^{2}\right) f_{g}^{(b)}\left(x_{3}, k_{2}^{2} ; M_{H} / 2\right) f_{g}^{(c)}\left(x_{4}, k_{4}^{2}\right) \sqrt{T_{s}\left(k_{2}, M_{H} / 2\right)},
$$

where we used, for the Green's function $G_{A}$, the approximation of the two-gluon exchange, see (16). Note that the Sudakov form-factor, $\sqrt{T_{s}\left(k_{2}, M_{H} / 2\right)}$, is present in the Green's function (shown explicitly in (22) $)$ and, implicitly, in the gluon distributions $f_{g}^{(b)}\left(x_{3}, k_{2}^{2} ; M_{H} / 2\right)$.

In this analysis we have introduced a cut-off, $k_{0}$, of the low momenta in the integration over $k_{1}$. The scale $k_{0}$ is meant to lie in the perturbative domain above the low hadronic scale of the proton form-factor. The structure of the nested integrations in (22) implies that this scale $k_{0}$ is a lower limit for all momenta. By studying the variation of the integrals (22) as a function of $k_{0}$, we obtain a measure of the sensitivity of the correction to poorly known details of the proton structure at low momentum scales. The strong coupling constant, $\alpha_{s}$, is kept fixed, as the running coupling introduces only logarithmic corrections, and, for the moment, we are interested in tracing only the leading power of $k_{0}$ that arises from the integral (22). In our estimate we use the Sudakov form-factor in the double logarithmic approximation:

$$
T_{s}(k, \mu)=\exp \left(-\frac{3 \alpha_{s}}{4 \pi} \log ^{2}\left(\mu^{2} / k^{2}\right)\right) .
$$

Let us start by performing the analysis of $Q_{R}$ with the conservative assumption of a vanishing anomalous dimension of the gluon density (i.e. a constant behaviour of the gluon density as a function of momentum). Then the integral over $k_{2}$ behaves in the same way as the integral defining the Two Pomeron Fusion amplitude. It is dominated by a contribution from a saddle point at a perturbative value, $k_{2}=\left(M_{H} / 2\right) \exp \left(-2 \pi / 3 \alpha_{s}\right)$, with only marginal sensitivity to the infrared domain.

The nested integrations over $k_{1}$ and $k_{4}$ in (22) are dominated by momenta close to the cut-off scale $k_{0}$ and lead to a result $\sim 1 / k_{0}^{2}$. This means that our correction is not small, but the magnitude is sensitive to details of the gluon distributions $f_{g}^{(a)}$ and $f_{g}^{(c)}$ at low momenta, and hence, unreliable. At first sight, this seems to imply that the whole Ansatz of choosing, for the absorptive correction, a perturbative gluon ladder, cannot be justified.

A closer look, however, shows that this may not be the final answer. Namely, many arguments have been given that the gluon density at very small $x$ should saturate, i.e. it scales and depends upon the ratio $k^{2} / Q_{s}^{2}(x)$. Here $Q_{s}(x) \sim x^{-\lambda}$ is the saturation scale which grows in $1 / x$ with $\lambda \simeq 0.3$. This leads to a substantial change of the $k^{2}$-dependence of the gluon density for momenta being smaller than the saturation scale: the saturation scale becomes an effective lower cut-off of the gluon momenta in the small- $k^{2}$ region. The phenomenological analysis of HERA data [36, 37] indicates that $Q_{s}^{2}\left(x_{4}\right)$ takes values of a few $\mathrm{GeV}^{2}$ for the range of $x_{4}$ relevant in our problem where $\log \left(1 / x_{4}\right)$ can be as large as 14 to 19 units. 
Applying these arguments to our integral (22) we find that, in the presence of the saturation scale, the momentum dependence of gluon distribution $f_{g}^{(c)}$ is modified, and the nested integrals over $k_{1}$ and $k_{4}$ give

$$
\int_{Q_{s}^{2}\left(x_{4}\right)} \frac{d k_{4}^{2}}{k_{4}^{4}} \int_{k_{0}^{2}}^{k_{4}^{2}} \frac{d k_{1}^{2}}{k_{1}^{2}} \sim \frac{\log \left(Q_{s}^{2}\left(x_{4}\right) / k_{0}^{2}\right)}{Q_{s}^{2}\left(x_{4}\right)} .
$$

With $Q_{s}\left(x_{4}\right)$ being well above the infrared scale $k_{0}^{2}$, this represents a well-behaved result with a low sensitivity to the infrared physics. Keeping in mind that, in (22), the integration over $k_{2}$ is infraredsafe, one finds that $Q_{R}$ is infrared stable.

An analogous reasoning can be applied also to the contribution of the virtual correction part of the triple Pomeron vertex to the integrals in (18). The behavior is approximated by:

$$
Q_{V} \sim \int_{k_{0}^{2}} \frac{d k_{1}^{2}}{k_{1}^{4}} \int_{k_{1}^{2}} \frac{d k_{2}^{2}}{k_{2}^{4}} \log \left(k_{2}^{2} / k_{1}^{2}\right) f_{g}^{(a)}\left(x_{2}, k_{1}^{2}\right) f_{g}^{(b)}\left(x_{3}, k_{1}^{2} ; M_{H} / 2\right) f_{g}^{(c)}\left(x_{4}, k_{2}^{2}\right) \sqrt{T_{s}\left(k_{1}, M_{H} / 2\right)}
$$

and a similar term, $Q_{V}^{\prime}$, is obtained by interchanging $f_{g}^{(b)}\left(x_{3}, k_{1}^{2} ; M_{H} / 2\right) \rightarrow f_{g}^{(b)}\left(x_{3}, k_{2}^{2} ; M_{H} / 2\right)$ and $f_{g}^{(c)}\left(x_{4}, k_{2}^{2}\right) \rightarrow f_{g}^{(c)}\left(x_{4}, k_{1}^{2}\right)$. One finds that the integral over $k_{2}\left(k_{1}\right)$ in $Q_{V}\left(Q_{V}^{\prime}\right)$ is stabilized in the infrared by the saturation scale, and the integral over $\boldsymbol{k}_{1}\left(\boldsymbol{k}_{2}\right)$ is stabilized by the Sudakov form-factor.

Collecting the estimates for $Q_{R}, Q_{V}$ and $Q_{V}^{\prime}$ one concludes that the perturbative determination of the amplitude of screening correction is possible provided that the gluon distribution $f_{g}^{(c)}\left(x_{4}, k_{4}^{2}\right)$ possesses the saturation property, i.e. the simple gluon ladder that we have used initially has to be corrected. In this case the momentum scale at the upper end of the screening correction, $f_{g}^{(c)}$, is not small, and our perturbative Ansatz is justified. Note that, in this context, saturation plays a role quite analogous to the Sudakov form factor at the production vertex: without the Sudakov form factor the momentum integral near the production vertex would not have been infrared-safe. Similarly, saturation renders the nested $k_{1}$ and $k_{4}$ integrations infrared-safe.

\section{Nonlinear evolution of the gluon distribution}

In this section we use the simplest available tool, the nonlinear Balitsky- Kovchegov (BK) equation 31, 32, 33, in order to model the unitarity corrections for the screening Pomeron ladder in Fig. 4, This equation resums fan diagrams of BFKL Pomerons (Fig. 5) in the LL1/x approximation, and it has been derived, in the large $N_{c}$ limit, for the case of a small probe scattering off a large nucleus. The BK equation generates, in a natural way, the saturation scale growing exponentially with rapidity [29, 35]. The equation has originally been derived for the dipole scattering amplitude, but later on it was reformulated as an explicit equation for the evolution of the unintegrated gluon distribution [48, 49], and in this paper we employ the latter form.

The hard rescattering correction amplitude in (18) was given in the momentum representation, in order to match the variables of the triple Pomeron vertex. In this section, however, we find it more 


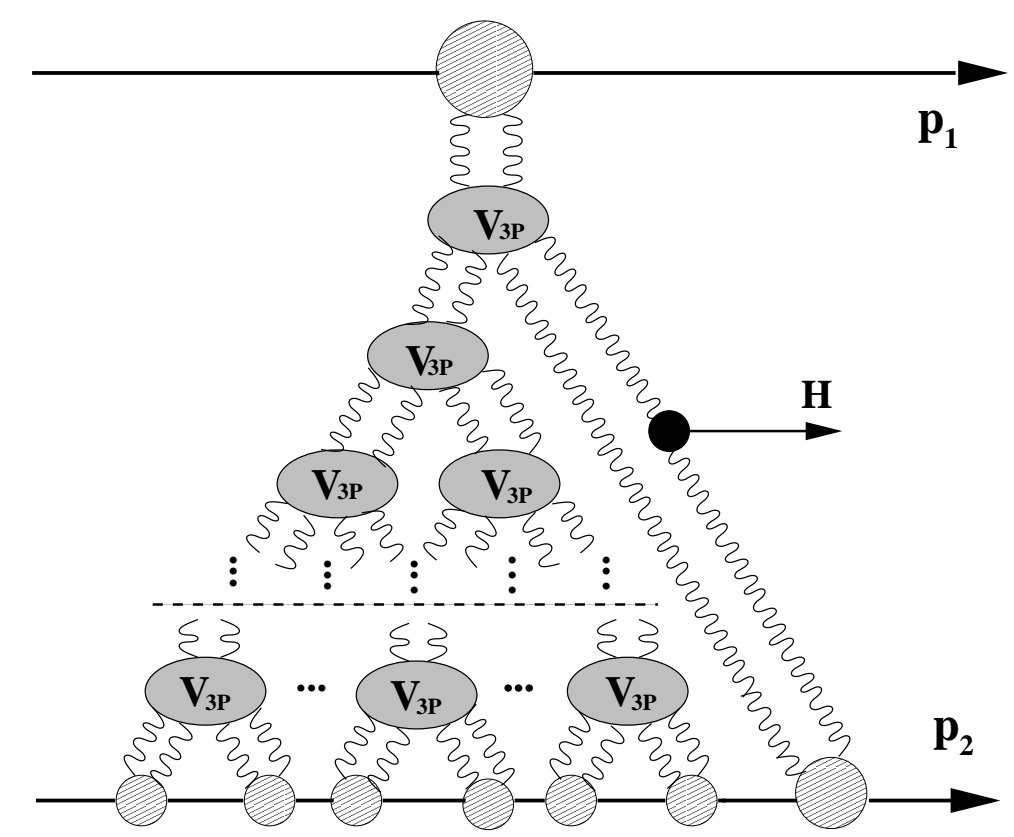

Figure 5: The fan diagram approximation to the hard rescattering correction to the exclusive Higgs boson production.

convenient to do a partial Fourier transform from momentum transfer carried by the Pomerons to the impact parameter variable. The impact parameter space seems to be advantageous, both in performing the non-linear gluon density evolution and for evaluating of the soft gap survival factor. The correct treatment of the impact parameter dependence of the scattering amplitude is difficult in the BK framework. In the original formulation of the BK equation, the gluons are assumed to be massless, in accordance with perturbative QCD; this contradicts, however, essential features of non-perturbative QCD dynamics leading to the confinement of colour. In particular, in the perturbative framework one finds contributions from large dipoles which generate a power like tail of the impact parameter profiles [50, 51]. Such a tail leads to a violation of the Froissart bound [52]. Therefore, the limit of large impact parameters of the BK must be modified, using some phenomenological model. In practice, all the applications of the BK equation to the proton structure are based on the approximation of treating the evolution at fixed impact parameter [35, 48, 53, 54, i.e. the evolution proceeds independently at each value of impact parameter $\boldsymbol{b}$.

Thus, our starting point is the form of BK equation proposed in Ref. [48]:

$$
\begin{gathered}
\frac{\partial f_{g}\left(x, k^{2}\right)}{\partial \log 1 / x}=\frac{\alpha_{s} N_{c}}{\pi} k^{2} \int_{k_{0}^{2}} \frac{d k^{\prime 2}}{k^{\prime 2}}\left\{\frac{f_{g}\left(x, k^{\prime 2}\right)-f_{g}\left(x, k^{2}\right)}{\left|k^{\prime 2}-k^{2}\right|}+\frac{f_{g}\left(x, k^{2}\right)}{\left[4 k^{\prime 4}+k^{4}\right]^{\frac{1}{2}}}\right\} \\
-\alpha_{s}^{2}\left(1-k^{2} \frac{d}{d k^{2}}\right)^{2} \frac{k^{2}}{R^{2}}\left[\int_{k^{2}}^{\infty} \frac{d k^{\prime 2}}{k^{\prime 4}} \log \left(\frac{k^{\prime 2}}{k^{2}}\right) f_{g}\left(x, k^{\prime 2}\right)\right]^{2},
\end{gathered}
$$

where the assumption has been made that the distribution of gluons is uniform inside a transverse disc with the radius $R$. The unintegrated gluon density in the impact parameter plane therefore takes 
the form:

$$
\tilde{f}_{g}\left(x, k^{2}, b\right)=f_{g}\left(x, k^{2}\right) \frac{\theta(R-b)}{\pi R^{2}} .
$$

In (26), the factor $1 / R^{2}$ in front of the non-linear term is a result of the impact parameter integration:

$$
\int d^{2} b \tilde{f}_{g}\left(x, k_{1}^{2}, b\right) \tilde{f}_{g}\left(x, k_{2}^{2}, b\right)=\frac{1}{\pi R^{2}} f_{g}\left(x, k_{1}^{2}\right) f_{g}\left(x, k_{2}^{2}\right) .
$$

In our case, however, it is necessary to control the impact parameter dependence in more detail. Therefore we assume, at $x_{0}=0.01$, an initial Gaussian impact parameter profile:

$$
\tilde{f}_{g}\left(x_{0}, k^{2}, b\right)=f_{g}\left(x_{0}, k^{2}\right) \frac{\exp \left(-b^{2} / R^{2}\right)}{\pi R^{2}},
$$

and we set the radius $R \simeq 2.83 \mathrm{GeV}^{-1}\left(R^{2}=8 \mathrm{GeV}^{-2}\right)$, in accordance with the proton radius seen in the $t$-dependence of the exclusive $J / \psi$ production at HERA. The evolution equation for the density of the unintegrated gluon reads:

$$
\begin{gathered}
\frac{\partial \tilde{f}_{g}\left(x, k^{2}, b\right)}{\partial \log 1 / x}=\frac{\alpha_{s} N_{c}}{\pi} k^{2} \int_{k_{0}^{2}} \frac{d k^{\prime 2}}{k^{\prime 2}}\left\{\frac{\tilde{f}_{g}\left(x, k^{\prime 2}, b\right)-\tilde{f}_{g}\left(x, k^{2}, b\right)}{\left|k^{\prime 2}-k^{2}\right|}+\frac{\tilde{f}_{g}\left(x, k^{2}, b\right)}{\left[4 k^{\prime 4}+k^{4}\right]^{\frac{1}{2}}}\right\} \\
-\pi \alpha_{s}^{2}\left(1-k^{2} \frac{d}{d k^{2}}\right)^{2} k^{2}\left[\int_{k^{2}}^{\infty} \frac{d k^{\prime 2}}{k^{4}} \log \left(\frac{k^{\prime 2}}{k^{2}}\right) \tilde{f}_{g}\left(x, k^{\prime 2}, b\right)\right]^{2} .
\end{gathered}
$$

This evolution is local in $\mathbf{b}$, but due to the non-linear term, for $x<x_{0}$, the impact parameter dependence cannot be factorized out from the resulting unintegrated gluon density, $\tilde{f}_{g}\left(x, k^{2}, b\right)$.

It is clear, however, that the BK equation (26) needs many improvements to be applicable in phenomenology, in particular it is essential to take into account, in some approximate manner, the NLL1/ $x$ corrections to the linear BFKL kernel [55, 56]. Following the path proposed in series of papers [57, 48, 49, we include, in the linear evolution kernel, the following improvements:

- the consistency constraint [58, 59] that models an essential part of the NNL1/ $x$ correction to the BFKL kernel and realizes, approximately, also a resummation of the corrections to all orders;

- the non-singular part in the $x \rightarrow 0$ limit of the DGLAP splitting function is added;

- the DGLAP quark density evolution is also taken into account, and the evolution equation is coupled to the gluon density evolution using the DGLAP splitting functions;

- the strong coupling constant is running with the scale given by gluon virtualities in the BFKL ladder.

These modifications are consistent with the exact NLL1/ $x$ corrections to the BFKL kernel, and they impose the constraints on the evolution which follow from the DGLAP formalism in the collinear limit 60, 61, 62]. The implementation of these corrections has been described in detail in the recent papers 
[48, 49], and the final form of the equation can be found there as well. It is rather straightforward to add, on top of the modifications described above, the impact parameter dependence, in a manner very similar to (30). The normalisation of the input parameterisation for the gluon and quark distributions at high $x$ has been tuned to provide a good fit of the $F_{2}$ data 63, and the obtained distributions have been shown to give a good description of inclusive heavy quark production at HERA and at the Tevatron 64. A detailed description of the properties of the obtained partonic densities will be given in a separate paper 63 .

In our numerical calculations of the exclusive Higgs boson production (18) we will take $\tilde{f}^{(c)}\left(x, k^{2}, b\right)$ as being the solution to the collinearly improved, impact parameter dependent BK equation. Let us remind that the other two gluon distributions of (18), $\tilde{f}^{(a)}\left(x, k^{2}, b\right)$ and $\tilde{f}^{(b)}\left(x, k^{2}, b\right)$, are probed at rather large values of $x \sim 0.01$, and non-linear effects can safely be neglected. Instead, we apply the prescriptions (7) and (8), using with the CTEQ5 parameterisation for the collinear gluon distribution functions.

\section{$5 \quad$ Soft gap survival probability}

Thus far, we have determined the hard component of the amplitude for exclusive Higgs boson production. The condition to leave the protons intact in the production process, however, imposes constraints on the soft rescattering of the protons. The soft gap survival probability factor, $\hat{S}^{2}$, quantifies the probability that the protons emerge from the reaction intact despite possible soft rescatterings. The gap survival probability can conveniently be estimated in the impact parameter representation by employing, for instance, the two-channel eikonal model [20, 21]. Therefore, we now translate the exclusive Higgs boson production cross section into the impact parameter representation.

Let us start with a general discussion. We consider a scattering amplitude of an exclusive production in the impact parameter representation

$$
\tilde{M}\left(\boldsymbol{b}_{1}, \boldsymbol{b}_{2}\right)=\int \frac{d^{2} q_{1}}{(2 \pi)^{2}} \int \frac{d^{2} q_{2}}{(2 \pi)^{2}} M\left(\boldsymbol{q}_{1}, \boldsymbol{q}_{2}\right) e^{i \boldsymbol{q}_{1} \boldsymbol{b}_{1}} e^{i \boldsymbol{q}_{2} \boldsymbol{b}_{2}},
$$

where $M\left(\boldsymbol{q}_{1}, \boldsymbol{q}_{2}\right)$ is the amplitude given as a function of the transverse momenta. Then, the integrated cross-section

$$
\sigma=\frac{1}{16 \pi} \int \frac{d^{2} q_{1}}{(2 \pi)^{2}} \int \frac{d^{2} q_{2}}{(2 \pi)^{2}}\left|M\left(\boldsymbol{q}_{1}, \boldsymbol{q}_{2}\right)\right|^{2},
$$

can be expressed in the following way:

$$
\sigma=\frac{1}{16 \pi} \int d^{2} b_{1} \int d^{2} b_{2}\left|\tilde{M}\left(\boldsymbol{b}_{1}, \boldsymbol{b}_{2}\right)\right|^{2}
$$

or, alternatively,

$$
\sigma=\frac{1}{16 \pi} \int d^{2} b_{1} \int d^{2} b\left|\tilde{M}\left(\boldsymbol{b}_{1}, \boldsymbol{b}-\boldsymbol{b}_{1}\right)\right|^{2}
$$



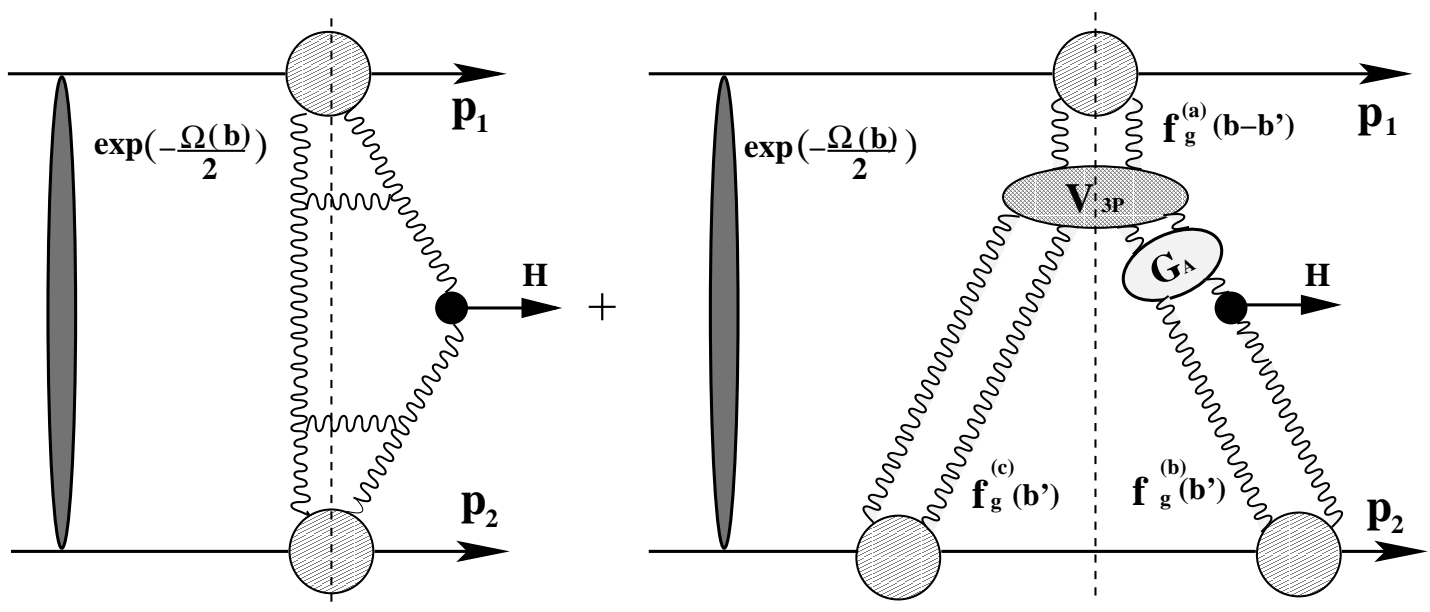

Figure 6: The exclusive $p p \rightarrow p H p$ amplitude with a hard and soft rescattering correction.

where $\boldsymbol{b}_{1}$ and $\boldsymbol{b}_{2}$ are transverse positions of the production vertex measured from centres of the protons, and $\boldsymbol{b}$ is the impact parameter of the collision.

In the Two Pomeron Fusion case, the off-diagonal unintegrated gluon distributions are probed at moderate values of $x \sim 0.01$ and at virtualities $k^{2}>1 \mathrm{GeV}^{2}$, so one can assume the gluon density to take a factorized form:

$$
\tilde{f}_{g}^{\text {off }}\left(x, x^{\prime}, k^{2}, b ; \mu\right)=f_{g}^{\text {off }}\left(x, x^{\prime}, k^{2} ; \mu\right) S(b)
$$

with

$$
S(b)=\frac{1}{\pi R^{2}} e^{-\frac{b^{2}}{R^{2}}}
$$

where $R \simeq 2.83 \mathrm{GeV}^{-1}$ is the proton radius, measured in the exclusive production of $J / \psi$ production at HERA (cf. our discussion of the impact parameter dependent BK equation), and $S(b)$ is normalized

$$
\int d^{2} b S(b)=1
$$

Therefore, the cross section for the production of the Higgs boson in the Two Pomeron Fusion approximation takes the form:

$$
\frac{d \sigma_{p p \rightarrow p H p}^{(0)}(y)}{d y}=\frac{1}{16 \pi} \int d^{2} b \int d^{2} b_{1}\left|S\left(\boldsymbol{b}_{1}\right) S\left(\boldsymbol{b}-\boldsymbol{b}_{1}\right) M_{0}(y)\right|^{2},
$$

with $M_{0}(y)$ given by (9). Including the hard rescattering correction, we arrive at the following expression:

$$
\frac{d \sigma^{(0+1)}(y)_{p p \rightarrow p H p}}{d y}=\frac{1}{16 \pi} \int d^{2} b \int d^{2} b_{1}\left|S\left(\boldsymbol{b}_{1}\right) S\left(\boldsymbol{b}-\boldsymbol{b}_{1}\right) M_{0}(y)+\tilde{M}_{\mathrm{corr}}\left(y, \boldsymbol{b}, \boldsymbol{b}_{1}\right)\right|^{2},
$$

where the dominant parts of both $M_{0}(y)$ and $\tilde{M}_{\text {corr }}\left(y, \boldsymbol{b}, \boldsymbol{b}_{1}\right)$ are imaginary, and

$$
\operatorname{Im} \tilde{M}_{\text {corr }}\left(y, \boldsymbol{b}, \boldsymbol{b}_{1}\right)=-\left\{\frac{9}{8} 16 \pi^{2}\left(2 \pi^{3} A\right) \int_{x_{a}}^{x_{b}} \frac{d x_{4}}{x_{4}} \int \frac{d^{2} k_{2}}{2 \pi k_{2}^{4}} \int \frac{d^{2} k_{3}}{2 \pi k_{3}^{4}} \int \frac{d^{2} k_{4}}{2 \pi k_{4}^{4}}\right.
$$




$$
\begin{aligned}
\times & {\left[V_{3 P} \otimes \tilde{f}_{g}^{(a)}\left(x_{1}, \boldsymbol{b}-\boldsymbol{b}_{1}\right)\right]\left(\boldsymbol{k}_{2}, \boldsymbol{k}_{4}\right) G_{\mathrm{A}}\left(\boldsymbol{k}_{2}, \boldsymbol{k}_{3} ; x_{1}, x_{2} ; M_{H} / 2\right) } \\
& \left.\times \tilde{f}_{g}^{(b)}\left(x_{3}, k_{3}^{2}, \boldsymbol{b}_{1} ; M_{H} / 2\right) \tilde{f}_{g}^{(c)}\left(x_{4}, k_{4}^{2}, \boldsymbol{b}_{1}\right)\right\}-\{y \rightarrow-y\} .
\end{aligned}
$$

The impact parameter dependence of $\tilde{f}_{g}^{(a)}$ and $\tilde{f}_{g}^{(b)}$ is given by $S(b)$ and may be factorized out, in analogy with the case of the Two Pomeron Fusion amplitude. The impact parameter dependence of $\tilde{f}_{g}^{(c)}$ is determined by the BK evolution, and it is different from $S(b)$. Note that, in (18), the convolution in the momentum transfer $\boldsymbol{q}$ leads to a product of $\tilde{f}_{g}^{(b)}$ and $\tilde{f}_{g}^{(c)}$, evaluated at the same point, $\boldsymbol{b}_{1}$, in the transverse position space.

Having defined $\tilde{M}_{\text {corr }}\left(y, \boldsymbol{b}, \boldsymbol{b}_{1}\right)$, it is straightforward to write down the cross section for the exclusive production, taking into account the soft rescattering

$$
\frac{d \sigma_{p p \rightarrow p H p}^{(0+1), \Omega}(y)}{d y}=\frac{1}{16 \pi} \int d^{2} b \int d^{2} b_{1}\left|S\left(\boldsymbol{b}_{1}\right) S\left(\boldsymbol{b}-\boldsymbol{b}_{1}\right) M_{0}(y)+M_{\mathrm{corr}}\left(y, \boldsymbol{b}, \boldsymbol{b}_{1}\right)\right|^{2} \exp (-\Omega(s, \boldsymbol{b})),
$$

where $\Omega(s, \boldsymbol{b})$ is the opacity of the $p p$ scattering at squared energy $s$ and impact parameter $\boldsymbol{b}$. In this paper we adopt the opacity factor of the two channel eikonal model proposed in [20, 21]. The gap survival probability reads:

$$
\hat{S}^{2}=\frac{\left(\frac{d \sigma_{p p \rightarrow p H p}^{(0+1), \Omega}(y)}{d y}\right)_{y=0}}{\left(\frac{d \sigma_{p p \rightarrow p H p}^{(0+1)}}{d y}\right)_{y=0}}
$$

The impact parameter profile of the rescattering correction amplitude (40) is different from the Two Pomeron Fusion case; thus the gap survival factor will take different values in those two cases.

\section{Results}

We have performed all numerical estimates of the amplitude and of the cross section for the central exclusive Higgs boson production, choosing $\mathrm{y}=0$, assuming the LHC energy, $\sqrt{s}=14 \mathrm{TeV}$, and taking, for the Higgs boson mass, $M_{H}=120 \mathrm{GeV}$. As we have stated before, in this paper our main goal is a general understanding of the hard screening mechanism and an estimate of its magnitude, rather than a precise determination of the cross section.

As a first step, we have considered the rescattering due to a single BFKL ladder. As expected, due to the long rapidity evolution of the BFKL amplitude this correction is large. As we have discussed before, since this single-ladder correction is highly sensitive to the infrared region, the precise numerical value of its magnitude is not reliable. From this part of our analysis, we therefore only conclude that this type of hard rescattering may potentially be large and, in any realistic estimate, unitarity corrections of the screening BFKL ladder have to be taken into account. 
In the next step of our numerical analysis, therefore, we have made use of the BK-corrected unintegrated gluon density, $f_{g}^{(c)}$. First, using (40), we have evaluated the hard rescattering amplitude as a function of the impact parameter, at fixed $x_{4}$. The contribution of momenta lower than $k_{0}=1 \mathrm{GeV}$ was assumed to vanish. The main reason for that is that the gluon densities are poorly known for gluon momenta lower than $k_{0}$, so is the behaviour of gluon propagators, and in that region one has to rely on uncertain extrapolations. On the other hand, with the integrand of (18) being positive, the application of the cut-off leads to a conservative estimate of the correction term. Still, the main contribution to the correction amplitude comes from the range of momenta around the saturation scale $Q_{s}\left(x_{4}\right)$.

Recall that, for the four-point gluon Green's function which connects the Higgs vertex with the triple Pomeron vertex, we consider two different scenarios - the simple two gluon exchange (16) and the BFKL Green's function in the diffusion approximation (17). The strong coupling constant was fixed at $\alpha_{s}=0.15$, both in the Green's function and in the triple Pomeron vertex. Such a choice is suitable for the LL BFKL Green's function, as the small $\alpha_{s}$ reduces both the Pomeron intercept and the diffusion coefficient, in line with the NLO corrections to the BFKL evolution. This value of $\alpha_{s}$ seems, however, to be too small for the triple Pomeron vertex: thus the estimate of the correction is conservative. Therefore, in the vertex, we shall consider also values of $\alpha_{s}$ other than our default choice of $\alpha_{s}=0.15$, while in the BFKL Green's function we always keep $\alpha_{s}=0.15$.

In Fig. [ 7 we present the total hard rescattering correction to the amplitude in the form of the ratio

$$
R_{y}\left(y_{1}\right)=\left[\frac{\left|d \tilde{M}_{\mathrm{corr}}\left(y, \boldsymbol{b}, \boldsymbol{b}_{1}\right) / d y_{1}\right|}{\left|\tilde{M}_{0}\left(y, \boldsymbol{b}, \boldsymbol{b}_{1}\right)\right|}\right]_{b=b_{1}=0, y=0},
$$

with the impact parameter dependent Two Pomeron Fusion amplitude being given by (cf. (9) and (38) )

$$
\tilde{M}_{0}\left(y, \boldsymbol{b}, \boldsymbol{b}_{1}\right)=M_{0}(y) S\left(\boldsymbol{b}-\boldsymbol{b}_{1}\right) S\left(\boldsymbol{b}_{1}\right) .
$$

Here $M_{0}(y)$ has also been evaluated with the infrared cut-off $k_{0}=1 \mathrm{GeV}$, and the off-diagonal unintegrated gluon distributions have been obtained from the CTEQ5 collinear gluon distribution. Note that we plotted the ratio as a function of the rapidity related variable, $y_{1}=\log \left(1 / x_{1}\right)$, instead of the less convenient variable $x_{4}$ (the relation between these variables is given by (19)). The variable $y_{1}$ has the meaning of a rapidity distance of the triple Pomeron vertex from the projectile proton, and it is related to the invariant mass of the rescattering intermediate state by $M_{X}^{2} \sim k_{0}^{2} \exp \left(y_{1}\right)$.

In Fig. 7 we compare the results, obtained with the two choices of Green's function and with the two values $\alpha_{s}=0.15$ and $\alpha_{s}=0.25$. The relative sign of the correction term is negative. From this figure we draw the following conclusions:

- The hard rescattering correction is the highest for the highest mass of the diffractive intermediate state. This is due to the strong suppression of the gluon density in the proton at large values 


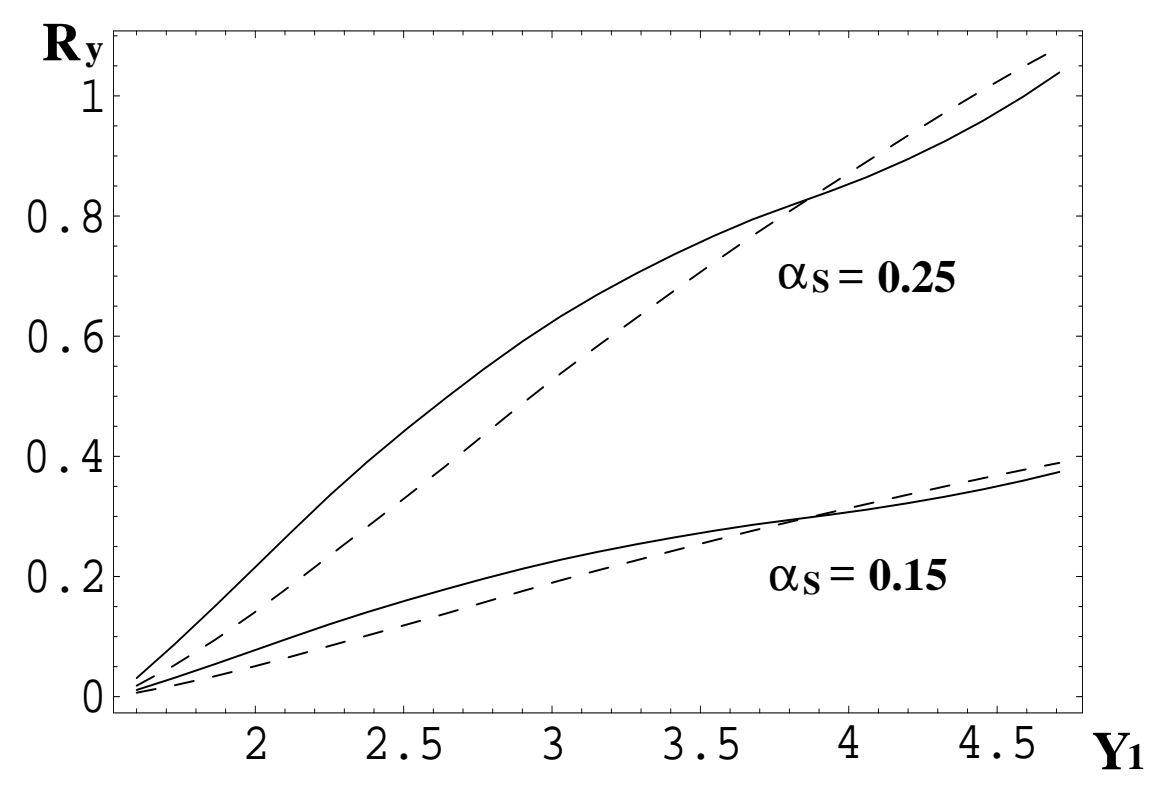

Figure 7: The ratio $R_{y}\left(y_{1}\right)$ (see 4 43)) of the hard rescattering correction to the Two Pomeron Fusion term at $b=0$, as a function of the rapidity distance, $y_{1}$, of the Triple Pomeron Vertex from the projectile protons, for $\alpha_{s}=0.15$ and $\alpha_{s}=0.25$ at $\sqrt{s}=14 \mathrm{TeV}$. The black line belongs to the Green's function, $G_{A}=G_{\mathrm{BFKL}}$, the dashed line to the approximation of two elementary gluons, $G_{A}=G_{0}$.

of the gluon $x$. We interpret this as an indication that the contribution of the hard rescattering correction is clearly separated from the soft rescattering contributions of the two-channel eikonal model [20, 21] which incorporates only the low mass diffractive intermediate states.

- There is no significant difference between the BFKL Green's function and the exchange of two elementary gluons. The main reason is that the BFKL evolution length is short for larger values of $y_{1}$, which give the dominant part of the corrections. The relative BFKL effects are more pronounced for small $y_{1}$, but this region provides only a small contribution.

- The relative magnitude of the correction is quite significant for $\alpha_{s}=0.15$, and for $\alpha_{s}=0.25$ the correction even exceeds the Two Pomeron Fusion amplitude. The interpretation of this striking result will be discussed in the next section.

In Fig. 8, results are shown for the impact parameter dependent correction amplitude, integrated over $y_{1}$, as a function of the transverse distance, $\boldsymbol{b}_{1}$, of the Higgs boson production vertex from the center of the target proton:

$$
R_{b}\left(b_{1}\right)=\left[\frac{\left|\tilde{M}_{\text {corr }}\left(y, b, b_{1}\right)\right|}{\left|\tilde{M}_{0}\left(y, b, b_{1}\right)\right|}\right]_{y=0, b=0} .
$$




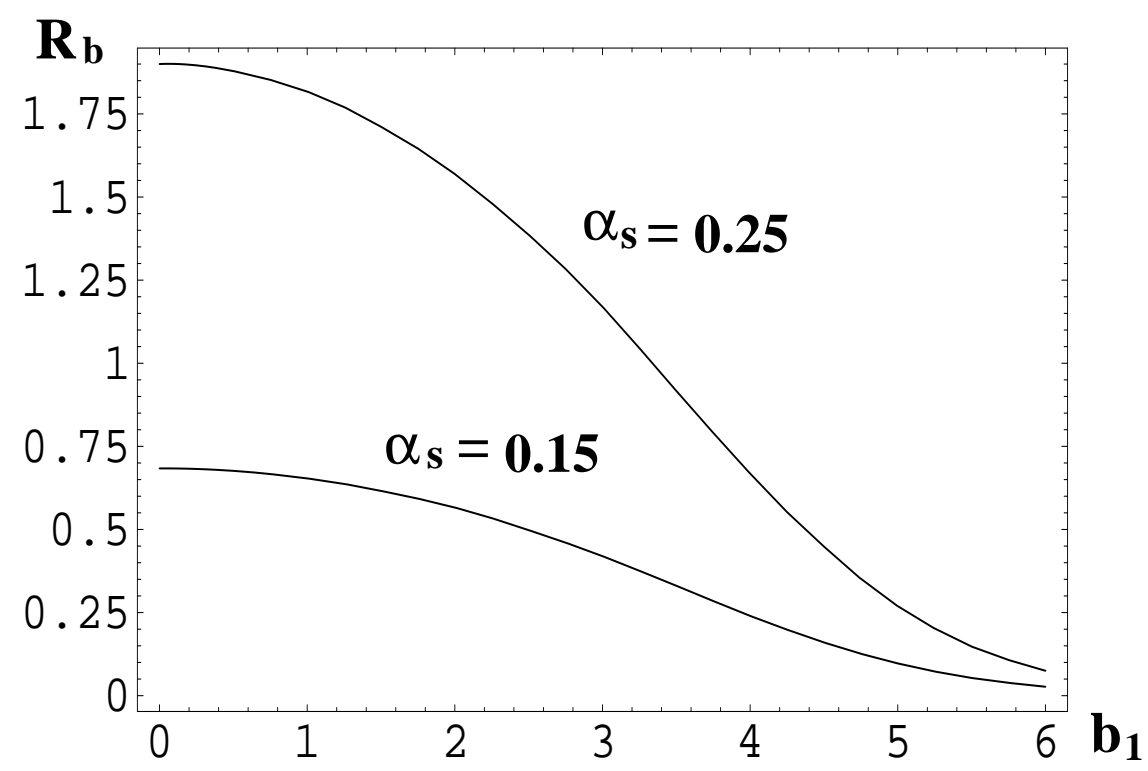

Figure 8: The ratio $R_{b}\left(b_{1}\right)$ (see 4 45)) of the hard rescattering correction to the Two Pomeron Fusion term at $b=0$, as a function of the transverse distance, $b_{1}$ of the Triple Pomeron Vertex from the centre of the target proton, for the two values $\alpha_{s}=0.15$ and $\alpha_{s}=0.25$ at $\sqrt{s}=14 \mathrm{TeV}$. We only show the results for the BFKL Green's function.

Here we have set $b=0$, but, in fact, in the ratio the impact parameter dependence coming from the form factor of the projectile proton drops out, and any value of $b$ can be taken. In Fig. 8 only the results obtained with the BFKL Green's function are displayed. There is not much difference between the BFKL Green's function and the two gluon approximation. One sees in the figure that the ratio of the absorptive correction profile, $\tilde{M}_{\text {corr }}\left(y, b=0, b_{1}\right)$, to the Two Pomeron Fusion profile, $\tilde{M}_{0}\left(y, b=0, b_{1}\right)$, decreases with increasing distance, $b_{1}$, from the proton center. Thus, the correction term tends to suppress the Higgs production inside the target proton. It is also clearly visible that the correction is large for both choices of $\alpha_{s}$.

Performing, in (41), the integrations over the transverse positions, $\boldsymbol{b}_{1}$ and $\boldsymbol{b}$, leads to the differential cross section of the exclusive Higgs boson production, $d \sigma_{p p \rightarrow p H p}^{(0+1), \Omega}(y) / d y$ which takes into account both the soft and the hard rescattering corrections. The results of this calculation, for various values of $\alpha_{s}$ in the triple Pomeron coupling, are collected in Tab. 1. At $\alpha_{s} \simeq 0.2$ the absolute value of the hard rescattering correction exceeds the Two Pomeron Fusion term, and this explains why, at this value of $\alpha_{s}$, the corrected cross section takes its smallest value. For all values of $\alpha_{s}$ that we have considered the cross section with the hard rescattering correction is smaller than the Two Pomeron Fusion cross section by factor larger than 2.5. Let us repeat that, by varying $\alpha_{s}$, we want to illustrate the sensitivity of the result to the size of the triple Pomeron coupling. Further discussion will be given in the next section. 


\begin{tabular}{|c|c|c|c|c|c|}
\hline$d \sigma_{\mathrm{TPF}} / d y$ & $d \sigma / d y\left(\alpha_{s}=0.15\right)$ & $d \sigma / d y(0.17)$ & $d \sigma / d y(0.2)$ & $d \sigma / d y(0.22)$ & $d \sigma / d y(0.25)$ \\
\hline 0.4 & 0.16 & 0.12 & 0.042 & 0.08 & 0.14 \\
\hline
\end{tabular}

Table 1: The differential cross section $\left(\frac{d \sigma_{p p \rightarrow p H p}}{d y}\right)_{y=0}$ (in fb), obtained from the Two Pomeron Fusion amplitude, and corrected by the hard rescattering and by the soft gap survival probability factor, for various values of $\alpha_{s}$ at $\sqrt{s}=14 \mathrm{TeV}$.

\begin{tabular}{|c|c|c|c|c|c|}
\hline$\hat{S}^{2}(\mathrm{TPF})$ & $\hat{S}^{2}\left(\alpha_{s}=0.15\right)$ & $\hat{S}^{2}(0.17)$ & $\hat{S}^{2}(0.2)$ & $\hat{S}^{2}(0.22)$ & $\hat{S}^{2}(0.25)$ \\
\hline 0.024 & 0.037 & 0.061 & 0.051 & 0.035 & 0.016 \\
\hline
\end{tabular}

Table 2: The soft gap survival probability factor, $\hat{S}^{2}$, for the Two Pomeron Fusion amplitude, corrected by the hard rescattering, for different values of $\alpha_{s}$ at $\sqrt{s}=14 \mathrm{TeV}$.

Numerical values of the survival probability, based on (42), are given in Tab. 2 for different values of $\alpha_{s}$. The variation of $\hat{S}^{2}$ with $\alpha_{s}$ is a result of the modification, due to the correction term, of the shape in impact parameter $b$ of the hard amplitude.

The definition of the survival probability, (42), which we used is different from the definition of $\hat{S}^{2}$ in [5]-11], 20, 21]. In the case when we consider only the Two Pomeron Fusion term and the Gaussian form of the form factors, the definitions of $\hat{S}^{2}$ in (42) and in Ref. 20] lead to the same numerical result. In the case of a general shape of the form-factors, however, we find it necessary to use the general formula for the soft gap survival factor of (40), (41) and (42).

\section{Discussion and future strategies}

The hard rescattering correction to the Two Pomeron Fusion amplitude, even after including unitarization of the single BFKL exchange, was found to be large. In particular, for a more realistic choice of the strong coupling constant, $\alpha_{s}=0.25$, in the triple Pomeron vertex, the correction exceeds significantly the Two Pomeron Fusion term. As a consequence, we have to conclude that we have 
found another potential source of a significant suppression of the exclusive Higgs boson production at the LHC.

As we have said before, we cannot consider our results as representing a reliable numerical final answer. In our calculation we have considered only a sub-class of the screening corrections which in a BFKL-based description of diffractive Higgs production - should be included, and in evaluating these diagrams, we have made severe approximations. In this paper, we have restricted ourselves to Pomeron fan diagram configurations in the exchanged screening system which lead to the BalitskyKovchegov evolution of the gluon density. Such a choice is a natural starting point for an estimate of the amplitude beyond the Two Pomeron Fusion contribution, but it does not exhaust the full correction, and, in future steps, more complex topologies of the interacting Pomerons have to be considered. Moreover, we have approximated the BK evolution kernel to be local in the impact parameter plane. In momentum space, this amounts to restricting all Pomerons to carry zero momentum transfer: such an approximation can be justified for the scattering on a sufficiently large nucleus; in the case of the proton, however, it looks somewhat questionable. Finally, we neglected the contribution from low mass excitations of the target proton in the intermediate state, which somewhat reduces the estimated magnitude of the rescattering correction.

Returning to the selection of diagrams, in our previous choice of contributions one recognizes a strong asymmetry between projectile and target protons, and it is obvious that a whole class of contributions is missing. In physical terms, the Pomerons that give rise to the BK fan diagrams propagate in the colour field of the projectile proton and should be screened, too (examples are shown in Fig. 9). It is expected that these new corrections will continue to come with alternating signs, i.e. the first screening correction of the screening Pomeron enters the amplitude with the positive sign etc. Subsequent iterations of this kind lead to a whole tower of corrections. For the total cross-section of nucleus-nucleus collisions an effective theory of interacting BFKL Pomerons [41, 42, 43, beyond the fan diagram approximation, has been formulated. Imposing a semiclassical approximation that resums Pomeron diagrams exemplified in Fig. 97, and restricting all Pomerons to have zero momentum transfer, numerical solutions have been found.

All these corrections can be derived from an effective action for interacting Pomerons of Refs. 41, 42, 43. Thus, a natural continuation of the present study could be an analysis of the hard rescattering in this framework. At present an evaluation of the interacting Pomeron diagrams of the type exemplified in Fig. 9a, analogous to the treatment of nucleus-nucleus scattering, might be realistic, whereas the diagrams with closed Pomeron loops (see Fig. 9b) are, to our understanding, still beyond reach. Nevertheless, a careful treatment of the effective interacting BFKL Pomeron field theory in the semi-classical limit should be capable to provide a more reliable estimate of the absorption of the hard gluonic ladders than the one performed in the present paper.

An important point in our analysis is the role of the saturation scale. Up to now, the analysis of diffractive Higgs production had been based upon a rather strict separation between hard and 
a)

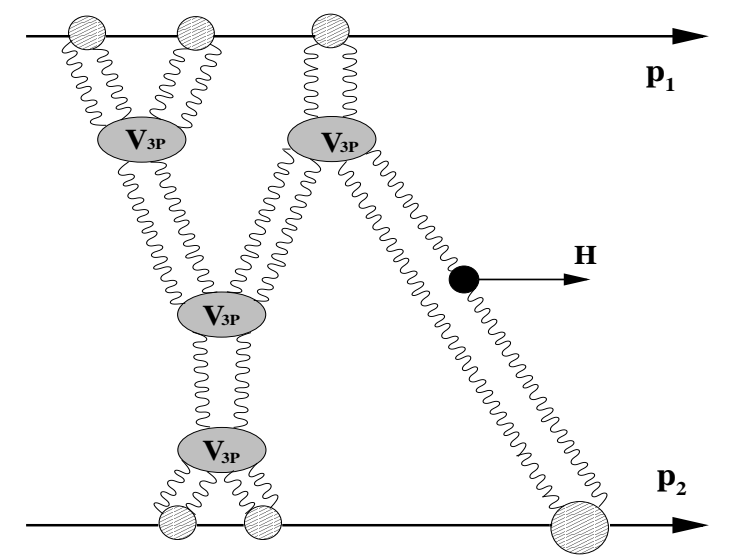

b)

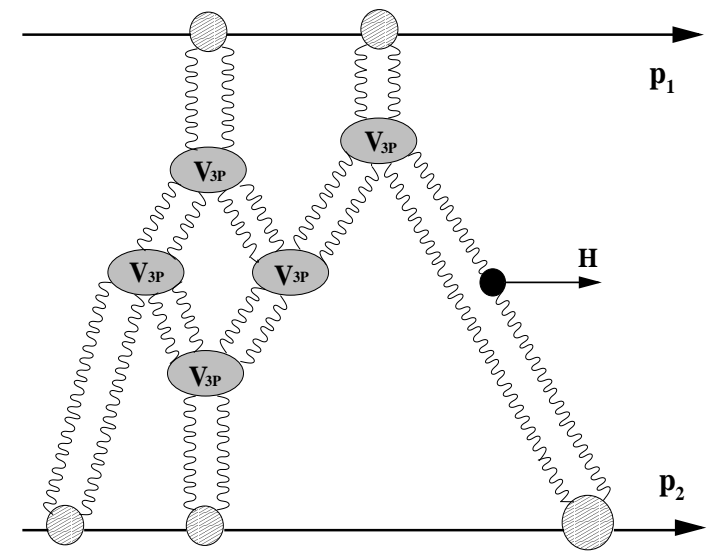

Figure 9: Examples of diagrams of interacting BFKL Pomeron field theory that contribute to the exclusive Higgs boson production: a) a diagram, without closed Pomeron loops, belonging to the class of diagrams that were studied in Refs. 41, 42, 43] in the case of nucleus-nucleus scattering; b) a diagram with a closed Pomeron loop.

soft contributions: the hard Two Pomeron Fusion amplitude and the soft rescattering, encoded in the survival probability factors. In the first part of our hard rescattering analysis, where a single BFKL Pomeron had been considered, we found a strong sensitivity to the infrared scale $k_{0}$ which, at first sight, seemed to invalidate the BFKL Ansatz for the rescattering. However, the existence of a saturation scale, which for $x$ values being as small as $x \approx 10^{-6}$ lies above the infrared region, removes this dominance of the small-momentum region and hence supports our perturbative approach. In other words, for the production of a Higgs boson which, at the production vertex, provides the large momentum scale of $M_{H}$, the perturbative nature of the scattering amplitude seems not be limited to the production vertex but seems to 'spread out' further away into the rescattering part of the amplitude.

\section{Summary}

In this paper we have investigated hard rescattering corrections to the exclusive Higgs boson production at the LHC. In a first step, the absorptive correction was given by the exchange of a single BFKL ladder which couples to the hard gluonic ladder through the triple Pomeron vertex. This amplitude has turned out to be strongly sensitive to the infrared region. In our main part, we then have replaced the single BFKL ladder by a Balitsky-Kovchegov gluon density. Due to emergence of a saturation scale in the perturbative domain, the infrared behavior stabilizes. We have found that the dominant contribution to the hard rescattering correction comes high mass diffractive excitations of the the projectile proton, indicating a clear separation of this contribution from that of low mass intermediate states of the standard soft rescattering. 
The correction was found to be negative, with the absolute value being close or even exceeding the leading Two Pomeron Fusion contribution. This implies that the effect is significant. However, a more precise estimate of the cross sections is not possible until yet more screening corrections are evaluated and certain approximation can be lifted. Nevertheless, our results seem to question the simple picture of the $p p \rightarrow p H p$ process, being described by two hard gluonic ladders that fuse into the Higgs boson and leaving space only to a soft rescattering of the protons. We have also discussed possibilities of improving, within the framework of the interacting BFKL Pomeron field theory, the description of the exclusive Higgs production cross section.

In order to arrive at a numerical estimate of the exclusive Higgs boson production cross section at the LHC, we have calculated the soft gap survival probability for the scattering amplitude containing both the Two Pomeron Fusion term and the rescattering correction. For this we have used the dependence of the amplitude on the transverse position; due to the different shapes of the Two Pomeron Fusion term and of the correction, the gap survival factors were found to depend on the strength of the triple Pomeron coupling.

In our final numerical estimate of the exclusive Higgs boson production cross section we found that the cross section may be smaller, and its theoretical uncertainty may be larger than previously expected.

The method developed in this paper may be also applied to other exclusive diffractive production processes in $p p$ or $p \bar{p}$ collisions at the Tevatron or at the LHC. As examples, let us mention the exclusive production of di-jets or the exclusive production of heavy scalar mesons in the central region. We also view the results of this paper as a step towards developing a better understanding of the effective field theory of interacting BFKL Pomerons and of its applications to high energy $p p$ and $p \bar{p}$ collisions.

\section{Acknowledgments}

We thank Markus Diehl, Jeff Forshaw, Valery Khoze and Misha Ryskin for useful discussions. S.B. thanks the Minerva foundation for a fellowship, K.K. is supported by the Graduiertenkolleg Zukünftige Entwicklungen in der Teilchenphysik. L.M. gratefully acknowledges the support of the grant of the Polish State Committee for Scientific Research No. 1 P03B 02828.

\section{References}

[1] S. Eidelman et al. [Particle Data Group], Phys. Lett. B 592 (2004) 1.

[2] A. Schafer, O. Nachtmann and R. Schopf, Phys. Lett. B B249 (1990) 331. 
[3] A. Białas and P. V. Landshoff, Phys. Lett. B 256, 540 (1991).

[4] J. R. Cudell and O. F. Hernandez, Nucl. Phys. B 471 (1996) 471.

[5] V. A. Khoze, A. D. Martin and M. G. Ryskin, Phys. Lett. B 401 (1997) 330.

[6] V. A. Khoze, A. D. Martin and M. G. Ryskin, Eur. Phys. J. C14 (2000) 525.

[7] V. A. Khoze, A. D. Martin and M. G. Ryskin, Eur. Phys. J. C24 (2002) 581.

[8] A. De Roeck, V. A. Khoze, A. D. Martin, R. Orava and M. G. Ryskin, Eur. Phys. J. C C25 (2002) 391.

[9] V. A. Khoze, A. D. Martin and M. G. Ryskin, Eur. Phys. J. C 26 (2002) 229.

[10] A. B. Kaidalov, V. A. Khoze, A. D. Martin and M. G. Ryskin, Eur. Phys. J. C 31 (2003) 387.

[11] A. B. Kaidalov, V. A. Khoze, A. D. Martin and M. G. Ryskin, Eur. Phys. J. C 33 (2004) 261.

[12] D. Kharzeev and E. Levin, Phys. Rev. D 63 (2001) 073004.

[13] M. Boonekamp, R. Peschanski and C. Royon, Phys. Rev. Lett. 87 (2001) 251806; Nucl. Phys. B 669 (2003) 277 [Erratum-ibid. B 676 (2004) 493]; Phys. Lett. B 598 (2004) 243.

[14] R. Enberg, G. Ingelman, A. Kissavos and N. Timneanu, Phys. Rev. Lett. 89 (2002) 081801;

R. Enberg, G. Ingelman and N. Timneanu, Phys. Rev. D 67 (2003) 011301.

[15] A. Bzdak, Phys. Lett. B 615 (2005) 240.

[16] V. A. Khoze, A. D. Martin and M. G. Ryskin, Eur. Phys. J. C23 (2002) 311.

[17] V. A. Khoze, A. D. Martin and M. G. Ryskin, Eur. Phys. J. C 34 (2004) 327.

[18] K. Belotsky, V. A. Khoze, A. D. Martin and M. G. Ryskin, Eur. Phys. J. C 36 (2004) 503.

[19] E. Gotsman, E. Levin and U. Maor, Phys. Rev. D 60 (1999) 094011.

[20] V. A. Khoze, A. D. Martin and M. G. Ryskin, Eur. Phys. J. C 18 (2000) 167.

[21] A. B. Kaidalov, V. A. Khoze, A. D. Martin and M. G. Ryskin, Eur. Phys. J. C21 (2001) 521.

[22] S. Bondarenko and E. Levin, arXiv hep-ph/0511124.

[23] L. N. Lipatov, Sov. J. Nucl. Phys. 23 (1976) 338 [Yad. Fiz. 23 (1976) 642].

[24] E. A. Kuraev, L. N. Lipatov and V. S. Fadin, Sov. Phys. JETP 45 (1977) 199 [Zh. Eksp. Teor. Fiz. 72 (1977) 377]. 
[25] I. I. Balitsky and L. N. Lipatov, Sov. J. Nucl. Phys. 28 (1978) 822 [Yad. Fiz. 28 (1978) 1597].

[26] L. N. Lipatov, Phys. Rept. 286 (1997) 131.

[27] J. Bartels Z. Phys. C 60 (1993) 471.

[28] J. Bartels, M. Wüsthoff, Z. Phys. C 66 (1995) 157.

[29] M. A. Braun Eur. Phys. J. C 6 (1999) 321.

[30] J. Bartels, M. G. Ryskin and G. P. Vacca, Eur. Phys. J. C 27 (2003) 101.

[31] I. Balitsky, Nucl. Phys. B 463 (1996) 99.

[32] Y. V. Kovchegov, Phys. Rev. D 60 (1999) 034008.

[33] Y. V. Kovchegov, Phys. Rev. D 61 (2000) 074018.

[34] N. Armesto and M. A. Braun, Eur. Phys. J. C 20 (2001) 517.

[35] K. Golec-Biernat, L. Motyka and A. M. Staśto, Phys. Rev. D 65 (2002) 074037.

[36] K. Golec-Biernat and M. Wusthoff, Phys. Rev. D 59 (1999) 014017.

[37] K. Golec-Biernat and M. Wusthoff, Phys. Rev. D 60 (1999) 114023.

[38] S. Munier and R. Peschanski, Phys. Rev. Lett. 91 (2003) 232001.

[39] S. Munier and R. Peschanski, Phys. Rev. D 69, 034008 (2004).

[40] S. Munier and R. Peschanski, Phys. Rev. D 70, 077503 (2004).

[41] M. A. Braun, Phys. Lett. B 483 (2000) 115.

[42] M. A. Braun, Eur. Phys. J. C 33 (2004) 113.

[43] M. A. Braun, Phys. Lett. B 632 (2006) 297; hep-ph/0504002.

[44] A. G. Shuvaev, K. J. Golec-Biernat, A. D. Martin and M. G. Ryskin, Phys. Rev. D 60 (1999) 014015

[45] M. Kimber, A. D. Martin and M. G. Ryskin, Eur. Phys. J. C 12 (2000) 655.

[46] M. Kimber, A. D. Martin and M. G. Ryskin, Phys. Rev. D 63 (2001) 114027.

[47] Y. L. Dokshitzer, D. Diakonov and S. I. Troian, Phys. Rept. 58 (1980) 269.

[48] K. Kutak and J. Kwieciński, Eur. Phys. J. C 29, 521 (2003). 
[49] K. Kutak and A. M. Staśto, Eur. Phys. J. C 41, 343 (2005).

[50] A. Kovner and U. A. Wiedemann, Phys. Rev. D 66 (2002) 051502.

[51] K. Golec-Biernat and A. M. Staśto, Nucl. Phys. B 668 (2003) 345 [arXiv hep-ph/0306279.

[52] A. Kovner and U. A. Wiedemann, Phys. Lett. B 551 (2003) 311.

[53] E. Gotsman, E. Levin, M. Lublinsky and U. Maor, Eur. Phys. J. C 27 (2003) 411.

[54] E. Iancu, K. Itakura and S. Munier, Phys. Lett. B 590 (2004) 199.

[55] V. S. Fadin and L. N. Lipatov, Phys. Lett. B 429 (1998) 127.

[56] M. Ciafaloni and G. Camici, Phys. Lett. B 430 (1998) 349.

[57] J. Kwieciński, A. D. Martin and A. M. Staśto, Phys. Rev. D 56, 3991 (1997).

[58] B. Andersson, G. Gustafson and J. Samuelsson, Nucl. Phys. B 467 (1996) 443.

[59] J. Kwieciński, A. D. Martin and P. J. Sutton, Z. Phys. C 71 (1996) 585.

[60] G. P. Salam, JHEP 9807 (1998) 019.

[61] M. Ciafaloni and D. Colferai, Phys. Lett. B 452 (1999) 372.

[62] M. Ciafaloni, D. Colferai and G. P. Salam, Phys. Rev. D 60 (1999) 114036.

[63] K. Kutak and L. Motyka, in preparation.

[64] H. Jung, K. Kutak, L. Motyka and K. Peters, in the Proceedings of the HERA-LHC Workshop, hep-ph/0601013, p. 369. 